\title{
Spondyloepiphyseal dysplasia congenita
}

INSERM

\section{Source}

INSERM. (1999). Orphanet: an online rare disease and orphan drug data base.

Spondyloepiphyseal dysplasia congenita. ORPHA:94068

Spondyloepiphyseal dysplasia congenita (SEDC) is a chondrodysplasia characterized by disproportionate short stature, abnormal epiphyses and flattened vertebral bodies. 